\title{
Design of Ultra-Low-Power Smart Wearable Systems
}

\author{
Grégoire Surrel $^{\star}$, Francisco Rincón ${ }^{\dagger}$, Srinivasan Murali ${ }^{\dagger}$, David Atienza* \\ * Embedded Systems Laboratory (ESL), EPFL, Switzerland. \\ gregoire.surrel, david.atienza@epfl.ch \\ $\dagger$ SmartCardia Sàrl. Switzerland. \\ francisco.rincon, srinivasan.murali@smartcardia.com
}

\begin{abstract}
Latest progress in microelectronics have enabled a new generation of low cost, low power, miniaturized, yet, smart sensor nodes. This new generation of wearable sensor nodes promise to deploy automated complex bio-signals analysis. In this paper, we present INYU, a wearable sensor device for physical and emotional health monitoring. The device obtains key vital signs of the user, namely Electrocardiogram (ECG), respiration and skin conductance continuously. Using this information, INYU can deliver a novel real-time algorithm for on-line heart-beat classification and correction that relies on a probabilistic model to determine whether a heartbeat is likely to happen under certain timing conditions. Thus, using this algorithm INYU can quickly decide if a beat is occurring at an expected time or if there is a problem in the series (e.g., a skipped, an extra or a misplaced beat). This new algorithm has been integrated in the processing pipeline of automated Heart-Rate Variability (HRV) analysis, both for time-domain (RMSSD, SDNN) and frequencydomain (LF/HF) algorithms.
\end{abstract}

\section{INTRODUCTION}

With an aging society, there is a strong need for autonomous systems that can provide quick and continuous health-care of people. Such systems can monitor the vital parameters, such as the Electrocardiogram (ECG) of the person, at home as well as in outdoor environments, and quickly alert the doctor or emergency response team in case of health problems. With the advent of the smart phones, continuous health care delivery has become easier. Today, researchers have been developing vital parameter wearable sensing nodes that can use the mobile phone as a gateway (and display), and directly alert the doctor or hospital in case of a problem with the health condition. While continuous mobile health monitoring opens up new care delivery mechanisms, it also creates novel challenges.

Indeed major challenges in the design of such new nodes are their inherent resource-constrained nature, coupled with harsh operating conditions susceptible to different noise sources (e.g., muscle noise, movement artefact and electromagnetic interference) and stringent autonomy

This work has been partially supported by the ObeSense (no. 20NA21_143081) and BodyPoweredSenSE (no. 20NA21_143069) RTD projects evaluated by the Swiss NSF and funded by NanoTera.ch with Swiss Confederation financing. requirements, which require a robust and multi-modal approach for signal acquisition and processing.

In this paper we present a concrete case study to illustrate how to address these major challenges. In particular, we present a wearable device, INYU, that can obtain multiple bio-signals (ECG, respiration and skin conductance) and operate under very tight energy constraints. Moreover, it includes a new signal processing pipeline with on-line beat-error detection and correction system that can be used to detect and correct beat errors in the ECG signal, as part of complex automated cardiovascular analyses.

According to [1], [2], analyzing the Heart-Rate Variability (HRV) is key in cardiovascular analysis because the variation of the heart-rate contains indicators of current diseases or impending cardiac diseases. However, the algorithms need to work on long uninterrupted series of beats. This context is acceptable in a clinically controlled environment, but is not conceivable for non-invasive portable systems where the number of available leads is much smaller and the contact noise is much higher.

The increasing quality of electrocardiogram (ECG) recordings combined with better R-peaks detection algorithms improved the situation but having an on-line validation and correction of the R-R series is desirable because the HRV indices can be heavily affected by erroneous beats in the series [3]. The correction of ectopic beats is said to be key, regardless of the spectral estimation technique.

Using a probabilistic model of the heart-beats occurrences, it is possible to classify and correct each R-R interval (see Fig. 1) to detect certain situations [4]. This solution is more robust than ignoring problematic parts of the series that can skew the resulting HRV indices. When recordings are done in real-life with wearable sensors, motion artifacts are expected, and correcting the series before processing is a necessary step. An automated correction of the series enables real-time extraction of HRV indices for immediate feedback to the user.

The algorithm has been developed for the INYU device (see Fig. 2). The algorithm is implemented as part of a processing pipeline, wherein the ECG signal is acquired, noise filtered out and advanced HRV parameters and arrhythmia conditions detected. 


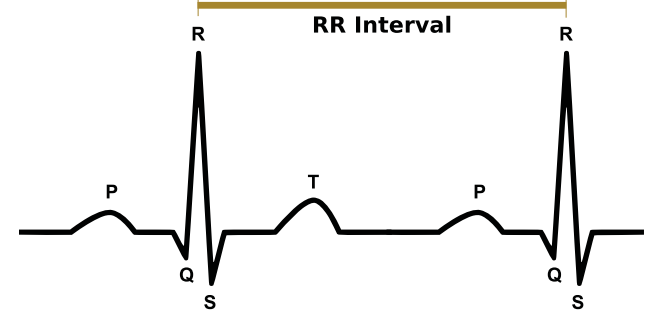

Fig. 1. Schematic diagram of normal sinus rhythm for a human heart as seen on ECG with the P-wave, QRS-complex and T-wave

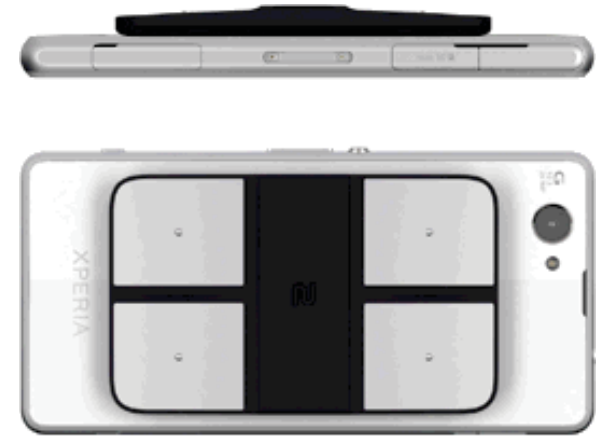

Fig. 2. The INYU device developed by SmartCardia can be put on the back of a phone. It implements several algorithms providing both low-level and high-level parameters about the holder. For instance, it can be the raw ECG, the current emotion or the stress level

\section{ECG Classification ALgORithm}

The reference algorithm is described in [4] where the R-R intervals between heart-beat events are modeled as following an Inverse Gaussian (IG) probabilistic distribution

$$
i g(x ; \mu, \lambda)=\sqrt{\frac{\lambda}{2 \pi x^{3}}} \times \exp \left(\frac{-\lambda(x-\mu)^{2}}{2 \mu^{2} x}\right)
$$

for $x=0$ where $\mu>0$ is the mean and $\lambda>0$ is the shape factor. The $\mu$ and $\lambda$ parameters are estimated by maximizing the log-likelihood applying an exponential decay over the recent history of recorded events. They are computed using the following equations [5]

$$
\hat{\mu}=\frac{\sum_{i=1}^{n} w_{i} R R_{i}}{\sum_{i=1}^{n} w_{i}}, \quad \frac{1}{\hat{\lambda}}=\frac{1}{n} \sum_{i=1}^{n} w_{i}\left(\frac{1}{R R_{i}}-\frac{1}{\hat{\mu}}\right)
$$

where $w_{i}=w\left(\tau_{i}\right)=e^{-\alpha \tau}$ which is the weighting function and $R R_{i}$ is the $i^{\text {th }}$ time interval between two beats.

There are four different situation which can be identified and corrected, which will change how the IG model will be used for beat-classification. For each beat, the four situations are estimated and get a score related to their likelihood. After a relative weighting the the individual scores, the highest one determines the final classification.

\section{Porting to INYU}

Different approaches where followed to have an efficient embedded algorithm with a lowered processing load in smart sensor nodes. Each modification was tested using the same process involving a training phase on one part the the Fantasia Database [6] and evaluating its performance on the remaining set of files. The main followed steps are:

1. Processing-window adjustment: the shorter the processing window, the lower the computational requirements. It also improves the response time of the system,

2. Removing non-critical operations: some operations improve results minimally but require a high processing load (e.g., exponential decay weighting),

3. Using others estimators: the $\mu$ and $\lambda$ parameters can be estimated using different approaches, which enables reducing CPU cycles by choosing another estimator if the results quality is not lowered,

4. Port to fixed-point: floating-point operations are not available on most micro-controllers, thus algorithms need to work well using fixed-point operations.

Once the algorithm was fully optimized by lowering the processing needs without degrading the performance, it has been integrated to the INYU embedded platform and added to the data-processing flow (see Fig. 3).

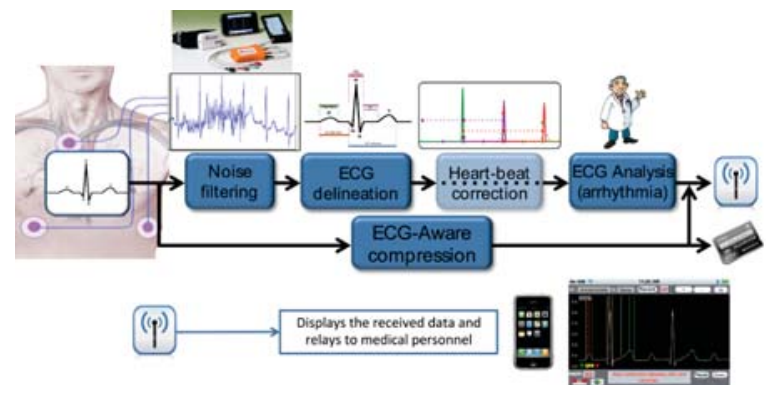

Fig. 3. The probabilistic heart-beat detection and correction algorithm was included in the processing pipeline as an additional step to improve automatic processing for HRV analysis, while still providing the original data for health professionals

\section{REFERENCES}

[1] J.T. Bigger, et al. Heart rate variability : Standards of measurement, physiological interpretation, and clinical use. Task force of the European Society of Cardiology and the North American Society of Pacing and Electrophysiology. Circulation, 93(5):1043-1065, 1996.

[2] U. Rajendra Acharya, et al. Heart rate variability: a review. MBEC, 44(12):1031-1051, 2006.

[3] G.D. Clifford, et al. Quantifying errors in spectral estimates of HRV due to beat replacement and resampling. TBME, 52(4):630638, 2005.

[4] L. Citi, et al.. A real-time automated point-process method for the detection and correction of erroneous and ectopic heartbeats. TBME, 59(10):2828-2837, 2012.

[5] M. C. K. Tweedie. Statistical properties of inverse gaussian distributions. AMS, 28(2):367, 1957.

[6] A. L. Goldberger, et al. PhysioBank, PhysioToolkit, and PhysioNet : Components of a new research resource for complex physiologic signals. Circulation, 101(23):e215-e220. 